\section{THE VALUE OF INOCULATION IN THE DIAGNOSIS AND TREATMENT OF ULCERS, AND DISCHARGES OF THE GENITAL ORGANS.}

By John C. Christophers, Esq., Surgeon, London.

LoNG antecedent to the time and writings of M. Ricord, inoculation had been practised by divers persons as a test for syphilis; but to that distinguished surgeon must be accorded the merit of detecting its real value, developing by its use many of the laws that appear to preside over the propagati.n of the venereal poison.

'The more frequently an experiment is repeated, and similar results are deduced by parties far removed from, and unknown to, each other, the stronger is the proof or the probability that such deductions are facts; and in this principle $I$ find an apology for the present paper.

The cases are selected from others of similar character, all going to prove the value of inoculation in the diagnosis and treatment of ulcers, and discharge invading the genital organs, and the majority to corroborate the experience and statements of M. Ricord: therefore they have little claim to originality or to novelty, but every claim to disinterestedness and to truth, and with the advantage that the latter pretension may be tested by all who will be at the pains to repeat the experiment. The principles and conclusions arrived at by $M$. Ricord are, no doubt, well known to all surgeons. It is not $\mathrm{mv}$ object to expound, but to corroborate them-a work which, I think, is not altogether useless, and one that $I$ know to be highly interesting.

In every case in which I have employed inoculation to test syphilis, and the sores have proved to be syphilitic, a specific ulcer has invariably resulted, and in no case (the inoculation failing) have secondary symptoms supervened, tending to prove the contrary. Howerer, it may be, and I believe it has been, argued, that it is not rational to produce disease to prove disease; but if it can be shown that no evils result from the practice, that important information may be gained, and that unnecessary annoyance and suspense may be saved, it appears not to be reasonable only, but feasible, benerolent, and imperative.

M. Ricord has stated (and I have seen many cases to corroborate the position) that provided a primary. sore of not more than four or five days' standing be effectually canterized or destroyed, (it being at that period but a local affection, the supervention of secondary symptoms may be averted thereby. 'Therefore chancres artificially produred may be submitted with safety to this practice, at the same time that they have been made to serve every purpose of diagnosis; for these sores, at a very early stage of existence, will bear unequivocal testimnny to the parent whence they spring. Furthermore, it does not appear that the number of chancres, even if left to thernselves, renders the supervention of secondary symptoms more certain, nor adds to their severity should they occur. Indeed, I have had occasion, several times, to observe the severity of the secondary affection existing in an inverse ratio to that of the primary disease.*

CAsE 1, shows the effect of inoculating with syphilitic virus; corroborates the experience of M. Ricord, that inoculating with matter taken from a chancre produces a sore of the same character.

twenty-one, of the lymphatic presented two sores on the glans penis resembling chaneres, and a bubo in the groin. Inoculated him on the arm nith the secretion from the sores. Two days elapsed, and a small angry-looking pimple appeared, which in three days became a sore of circular form, with a depressed, foul, sloughing centre, hard, elevated, and somewhat ragged edges, presenting an appearance identical with that whence the virus was obtained. The patrent was submitted to treatment. A month elapsed-the bubo had disappeared; the chancres on the glans had healed; the sore from the inoculation remained, though (by the repeated application of eanstic) it had lost its specific character, and was granulating rapidly. A week elapsed, and the sore had healed. I bad opportunities of seeing the patient for some weeks, during that period he was attacked by secondary symptoms.

CASE 2, shows the value of inoculation as a means of diagnosis; how a case, at first sight doubtful, is made clear and evident thereby.

aged twenty-tro, of the bilioso-sanguineons temperament, stated, that he had connexion with a suspected party six weeks and a few days previously, and not since then. The night before he consulted me, he perceived, for the first time, many small pustules around the corona glandis, and when I examined him, on the following morning, it was encircled with a crop of small, superficially excavated ulcers, with foul, depressed centres, eight or nine in number. My patient was most anxious to be informed of their true character, flattering himself, that the time that had elapsed since connexion freed him from the risk of contact. The case appeared somewhat doubtful,-1 - Ist, from the number of the sores; 2ndly, from their appearance; and 3rdly, from the length of time that had elapsed since connexion. To the two last, no great importance could be attached, as it is diffcult to discriminate between a chancre and a common ulcer in its earliest stage ; and cases have been recorded of chancres appearing so long as three months after connexion. To the first more weight was due. 'This, together with the extensive surface it would have been necessary to cauterize, caused me to forego this, my accustomed practice, and to propose inoculation, which was submitted to; and on the third day a pimple appeared, which wert on to form a chancre. My patient would submit to local treatment only, and in about three weeks the sores around the glans and that on the arm (the result of the inoculation) had healed, each leaving a very marked cicatrix. One month after this, he was attacked by severe pain in the posterior part of both knee-joints, accompanied by edema of the eyelids, syphilitic mottling, and urticaria-all readily succumbed to small doses of the hydriodate of potass. Some weeks elapsed, and he suffered anotler attack. Being at that time absent from town, he was directed by a practitioner to take three grains of blue pill every night; he became rapidly worse-cachectic ulcers appeared over the spine of the tibia on both legs. He returned to town, resumed the hydriodate of potass, and got rapidly well. About a month transpired, and another attack supervened, with ulceration of the tonsils; all again gave way to small doses of the hydriodate of potass. Since then he has suffered several accessions, each decreasing in severity, (which, indeed, appears to be the law in attacks of secondary syphilis, and all submitting to the same treatment. Some years have transpired since the last. He is now free from all disease, and from all evidence that such erer existed.

The value of inoculation as a means of diagnosis is evidenced by this case. The numerous sores led me to think they were not of syphilitic origin. Inoculation corrected the error, and the secondary symptoms that supervened proved the innculation. The secondary symptoms were trifling, indeed, (when not aggravated by mercury,) tending to show that the severity of secondary symptoms does not depend on the number of primary sores, (in this case they were eight or nine in number,) and is an argument in favour of the safety of producing a chancre artificially to test the existence of syphilitic disease.

CAsEs 3 and 10, show the effect of inoculating with the secretion from sores strictly resembling chancres situated on the glans penis, corroborating the experience of M. Ricord, that a non-syphilitic sore does not (by inoculation) produce a specific sore.

- aged forty-five years, suffering from retention of mymptoms indicative of stone in the bladder (having all the characters of chancres,) with enlarged inguinal glands. He declared he had not had connexion for eight months. Inoculated him on the arm with the secretion from the sores without producing any effect; the ulcers healed with waterdressing; the enlarged glands disappeared. I had opportunities of seeing him during many months; he was not attacked by secondary symptoms.

CASE 10.-A married man of industrious and steady habits, presented an enormous crop of ulcers, to all appearance chancres, completely encircling the glans penis and prepuce. His wifế was in perfect health. He declared he had not had connexion with any other party. He was inoculated on the arm several times, at intervals of from three to four days, with the secretion from the sores, without producing any effect. The sores healed rapidly under the influence of simple water-dressing. I saw him several years after this; he had not suffered from secondary symptoms.

I am confident the most practised eye would have failed in detecting the slightest shade of difference between the appearance of the sores in both these cases, and that of true chancres. Inoculation spared the patient, in both instances, much unnecessary suffering, moral and physical, and that without entailing the slightest annoyance: at the same time, it indicated the treatment.

CASE 5, shows the effect of inoculating with the secretion from a sore resembling a chancre, situate on the labium pudendum, the patient being under the full influence of mercury at the time. apparently a chanere, with a mucoso-purulent vaginal discharge of six weeks standing. She had been under treatment fourteen days, and stated she took ten grains of blue pill every night during that period. She was fully under the influence of mercury. 
She was inoculated with the secretion from the sore, and with the vaginal discharge; no effect was produced. Simple treatment was adopted; the sore healed, the discharge ceased, and no secondary affection supervened.

The sore presented all the characters of a true chancre, and the question therefore was mooted-Whether the circumstance of the patient being under the full influence of mercury at the time she was inoculated prevented the inoculation taking? in other words, Does mercury protect the constitution, or render the syphylitic virus inert?

Case 6, shows the effect of inoculating with matter taken from a bubo, the primary sore having healed some weeks previously, the patient being under the full influence of mercury at the time of inoculation.

, aged nineteen, presented a bubo in the left groin, with a cicatrice on the prepuce, which, he stated, marked the spot where a chancre had been, and which had healed some weeks previously. He was under the influence of mercury. 'The bubo was opened, and he was inoculated on the arm with the discharse; a pimple appeared two days afterwards, which assumed gradually the characters of a true chancre; the opening made into the bubo put on the same appearance. He continued the mercury; the sore artificially produced was cauterized, and in a month be was quite well.

In this case, when inoculation was practised, the patient was under the influence of mercury, and the primary sore bad healed; therefore it goes to show-1st, that mercury did not protect from, or destroy the effect of, the syphilitic virus; 2ndiy, that buboes may be produced by direct absorption; 3rdly, that buboes should be opened as early as posstble; for in this case the patient had been submitted to one course of mercury, whereby the disease was thought cured ; "when the necessity for a second course arrives, (at least, in the minds of those who treat syphilis by that means, inasinuch as the bubo contained syphilitic virus, therefore it possessed the power to induce an accession of primary sores. This case also corroborates the experience of MI. Ricord, who has succeeded frequently in producing specific sores with matter taken from buboes.

CAses 7 and 8 , show the effect of inoculating with the secretion of a phagedenic ulcer, resembling a chancre, situated on the glans penis; corroborating the opinion of $M$. Ricord, that there are not severai distinct syphilitic poisons, bat that the one poison is modified by idiosyncrasy of constitution, mode of life, situation, and treatment.

- - presented a phagedenic ulcer, (in appearance a chancre, situated on the glans penis, extending into the urethra, and of nine weeks standing. It occupied a large surface, deeply; it developed itself five days after connexion, and was treated by mercury, which appeared to aggravate it, and it became worse rapidly. He was inoculated on the arm; on the third day a pimple appeared, which became a well-developed chancre, and did not present any phagedenic character. At the time of inocalation, the patient was in a very weak state, though improvingevidently suffering more from the effect of mercury than from any other cause. $\mathrm{He}$ was ordered generous diet, pure air, and moderate exercise. The phagedenic chancre was canterized repeatedly; no medicine was administered, and in three weeks he was cured. Three months after this, he was attacked by secondary symptoms in a very mild form, which readily succumbed to small doses of hydriodate of potass.

CASE 8._-Presented a phagedenic ulcer (quite a chancre in appearance) situated on the glans penis, and a sore of the same character on the posterior part of the thigh; he admitted having had connexion with a suspected party, and five days afterwards he perceived a sore just within the prepuce; he did not interfere with it for a fortnight, when it became so painful he could not walk. He applied to a surgeon, who ordered mercury, till he salivated him; the sore on the prepuce healed, but a very large superficial sore appeared on the glans, which, with that on the posterior part of the thigh, went on to increase rapidly. They both presented the same characters. He stated he took two mercurial pills at riight, and one in the morning, during three months. He was so weak and reduced he could barely walk: he was ordered the hydriodate of potass, but it purged him so severely, it became necessary to discontinue it. He was fully under the influence of mercury. He was inoculated on the arm with the secretion from the phagedenic sore on the glans. Twenty-four hours after, a small pimple arose, which went on to increase, and became a well developed c'ancre; it did not present any of the phagedenic character of the sore whence it emanated, but solely that of true chancre. Its form was strict'y circular; its edges were red and indurated; its centre was excavated and foul: The patient's health was improving at the period of inoculation, on good air, generous diet, and the absence of medicine; the sores were cauterized repeatedly, and went on rapidly losing their specific chatacter, and granulating healthily. Three months elapsed, and he was quite cured. The repeated application of caustic, combined with good air and generous diet, worked his cure.

In the foregoing cases, both patients had been treated with mercury, and in both instances the general health failed, and the disease as rapidly increased. The mercury being omitted, and good air and generous diet, with local treatuent substituted, they both as rapidly got well. In each case, when inoculation was practised, the general health and the sores were improving, and it is to this fact $I$ attribute the circumstance that the artificial sores refused to assume the phagedenic character of those whence they emanated-a fact going to show, that the syphilitic poison is modified and controlled by the condition in which it finds the constitution at the time it is submitted to its influence-that is to say, the virus that would produce a benign chancre (the patient's general health being good) would be equally able to produce a phagedenic chancre, his general health being impaired.

CASE 9, shows the value of inoculation in the diagnosis of discharge from the urethra, having all the appearance of a gonorrhcea.

- presented a discharge from the urethra of long standing, and which had resisted all previous treatment. Inoculated him with the discharge on the arm. In two days a pimple developed itself, which went on to be a confirmed chancre. It was of circular form, with a sloughing centre, and a hard, red, extended base. No ulcer could be seen within the urethra, nor could any induration be detected : he took three grains of blue pill every night. The discharge speedily ceased; the inoculated part healed; and the patient was cured. Some months after he had an attack of secondary symptoms.

The value of inoculating, in this case, is too evident to require comment. It also corroborates cases related by $\mathrm{M}$. Ricord and others. Analogous cases occurred to Mr. John Hunter, which led, in great measure, (through false deduction,) to promulgate the idea that gonorrbea should be treated by mercury; inasmuch as he was thereby brought to conclude that the poison in syphilis and in gonorrhœa are identical.

CASE 10, shows that the pus of a gonorrhoea does not produce a specific sore by inoculation.

presented a gonorrhoea of many months' standing, which had resisted all kinds of remedies. Inoculated him on the arm with the discharge several times, at intervals of a few days : no effect was produced. No tenderness or induration of any part of the urethra could be detected. 'The stream of urine was slightly twisted, though its calibre was not diminished. Passed a catheter every other day for a month: this effected a permanent cure. I had opportunities of seeing him for several years: no secondary affection supervened.

Case 11 presented a gonorrhcea of long standing. No remedy appeared to control it. Inoculated him on the arm without effect. He was cured by the same means as the preceding patient.

I have never seen a specific sore produced by inoculating with gonorrhcal virus. I have seen, on one or two occasions, a small vesicle produced thereby; but the same appearance may be imitated by inserting a little milk under the skin, and can by no means be mistaken for a specific sore. M. Ricord states, that constitutional or secondary symptoms never succeed to a simple gonorrhcea, and that in those cases where secondary symptoms have been said to follow a simple gonorrhcea, they have been cases of undetected chancres within the urethra, and not cases of gonorrhcea at all.

Cases might be cited in which inoculation has been employed with the secretion from secondary syphilitic sores. I have witnessed this experiment more than once; in each case it tended to uphold the statement of M. Ricord, that secondary symptoms are not capable of reproduction by inoculation, though they may be transmitted from parent to child, of which the following case is an example.

Case 12. - - presented her infant, a few weeks old, covered with a copper-coloured eruption, and she (the mother) presented a well-developed chancre on the nipple of her breast. She stated, her husband had diseased her before she became pregnant, and that during the period of gestation she had an eruption somewhat similar to that on her infant, and with which it (the infant) was born. Soon after its birth, her nipple became painful, and each day it got worse. The child had not any sore about the mouth, lips, or fauces, that could be detected. The mother and infant were both submitted to mercurial treatment, and both as rapidly got well.

This is the only ease of the kind I have had the opportunity of seeing, and one in which it appeared that the venereal poison, in its primary form, was propagated by a secondary affection. 
Though, is it not probable that the mother had a chancre at the time of parturition, and that the lips, mouth, or fauces of the infant were inoculated therefrom, and that the effect of such inoculation had healed before the mouth was inspected, some weeks after its birth?

Taking a review of the foregoing cases, in which inoculations were practised, the retrospect points to the following facts: 1st, That in some, the inoculation alone furnished the only means of diagnosis. 2ndly, That in others it corrected it. Lastly, That in all, it tested, proved, or confirmed it, and that in a manuer at once decided and correct. That great prejudice exists against the employment of inoculation as the test for syphilis there can be little doubt, a feeling equally strong, at one time, was excited against the practice of vaccination, over which the increasing good sense of an enlightened age has happily triumphed; and as inoculation in the diagnosis and treatment of ulcers and discharge invading the genital organs comes to be more frequently practised, I feel confident its uses and advantages will be recognised, admitted, and proved, and that it will be thereby elevated to a position, in the estimation of a practical surgeon, that its inestimable value so eminently entitles it to hold.

Upper Montagu-street, Montagu-square, October 15th, 1845.

\section{THE PATHOLOGY OF BRIGHT'S DISEASE OF} THE KIDNEY.

By Richard Quain, M.D., House-Physician to University College Hospital.

Dr. JoHnson's communication to the Medico-Chirurgical Society has called attention to the pathology of albuminuria. It has occurred to me to have examined several specimens of kidneys, the secretion from which, during life, presented the characteristic which has given a designation to this disease. The microscopic observations which I have made have been noticed from time to time by Dr. Williams, in his Clinical Lectures, published in the Medical Gazette, and, taken in connexion with his own, have afforded grounds for the conclusions which he has there expressed. The observations referred to do not agree with the doctrines announced by Dr. Johnson, and the subject is of sufficient importance to require that a statement of the difference be made.

Dr. Johnson believes that an increase of the fat globules, existing in the cells within the uriniferous tubes is the morbid change which occurs in Bright's disease; that this change is the result of constitutional causes; and that this deposit is the cause, and not the result, of the congestion of the kidney, which finally leads to the presence of albumen in the urine. My object in this communication being rather to record my own observations than to disprove those of Dr. Johnson, I shall confine myself to a statement of the facts observed. In perhaps more than fifty specimens of this disease examined by me, the fatty condition of the kidney was in one instance only sufficient to attract much attention. The case is thus mentioned by Dr. Williams, in the Medical Gazette for August 1st, p. 577.*

"Dr. Quain examined some of the matter found in the cortical portion of Mayhew's kidneys, and it presented an unusual quantity of opaque globules, which are, in fact, fatty globules, and demonstrate that the cacoplastic deposit was here more fatty than usual."

I have, in many other instances, observed the presence of fat globules, but not to such an extent as to induce the belief that the presence of fat could be the only organic change which had taken place. In almost every case I found decided evidence of the presence of other deposits; of what Dr. Williams has so appropriately named cacoplastic (badly organizable) lymph, such as we find in or on other organs and tissues, which have been the seat of unhealthy inflammation, or degraded nutrition. This matter has been generally observed to assume-1. The form of nucleated cells, varying in size and shape, and also in the number and character of the nucleoli. 2. As simple granular matter, the nucleated cells being fewer in number. 3. In two instances I have observed the deposit to assume a distinct filamentous or fibrous character.t In the two first varieties fat globules have been noticed, as already stated, to a greater or less extent.

The seat of the deposit there was little difficulty in assigning to the substance of the kidney external to the tubes. In two recent instances, owing perhaps to improved experience in manipulating, I have been able to observe the granular matter contained within the tubes themselves.

If these observations are correct, Dr. Johnson has taken a limited view of the morbid condition of the kidney in this dis-

* This lecture was delivered May 8 th.
+ This observation is fully confirmed by those of Dr. Escholtz, which are translated in the last number of the Medical Gazette. ease. The examinations which $I$ have made, taken in connexion with those made by Dr. Williams, and his views as to the nature of this morbid deposit, as declared in his Principles of Medicine, but more particularly in reference to the present disease, in the clinical lecture already quoted from, lead to the following inferences as to the pathology of Bright's disease-viz., that it is the deposit of a badly organizable or cacoplastic matter in the cortical substance, and within the tubes of the kidney. This deposit generally takes place in an unhealthy system, as the result of congestion, produced either mechanically as by diseased heart, or by some irregularity in the function of the skin. The same effect may be produced by the circulation of stimulants, as alcohol, with the blood. Those conditions - diseased heart, perspiration suppressed by cold, or by experimentally coating the surface with substances, impermeable to this secretion, and intemperate habits, are constantly found associated with albuminuria. These facts, without entering on the causes of the disease, are mentioned with a view of showing that congestion precedes, rather than follows, the deposit in the kidney-a view confirmed by the results of treatment.

The deposits in the kidney assume the following characters:

1. The simple enlarged nottled kidney, the surface of which, on removing the capsule, is generally smooth. In this the deposit consists of simple nucleated cells, more or less mixed with granular matter. This form is analogous to the hypertrophied mottled liver.

2. The truly granular or atrophied kidney, the surface of which is rough, irregular, and generally of a pale-reddish colour. In this form, the filamentous tissue, contractile in its nature, as such formations always are, exceeds the quantity of the cellular or granular matter. The latter I have observed extending to the convoluted extremities of the tubes. The contractile tissue surrounding the tubes and bodies can be readily supposed to give rise to the rough or granular formation. This form resembles the hob-nailed or gin-liver.

3. The large, fabby, fatty-looking kidney. In this the quantity of fat exceeds the amount of the other matters present The fat is present in the substance, and probably, as shown by Dr. Johnson, in the tubes themselves. This resembles the fatty degeneration of the liver.

We have thus three principalforms which this disease has been generally found to assume: minor modifications of these may be produced by the relative proportions in which these deposits are present. There is no reason to infer that one condition is the necessary antecedent of another, but that the character assumed in the first instance may be permanent. A case which has recently been under Dr. Williams's care in this hospital, seems to confirm this view. The patient had been under observation during more than five years, and after death, the kidneys were found large, mottled, and non-granular. It proves, at least, that this form of mottled kidney is not necessarily an acute disease.

University College Hospital, November, 1845.

\section{UNIVERSITY COLLEGE MEDICAL SOCIETY.}

FRIDAY, NOVEMBer 14.-DR. R. DAWSON BarLING, PRESIDENT. Anatomy and Craniology.

A paper was read this evening on the "Anatomy of the Brain in Man and Animals, as applied to some of the leading doctrines in its Physiology, and especially to Craniology," by Mr. Jote Marshall.

The author commenced by alluding to the grey matter as the seat of power, and to the white fibres as serving the office of conductors, in the functions of the nervous system; and having referred at length to the parts supposed to be concerned in volition, sensation, in the emotional and reflex phenomena, and in the coordination of muscular movements, he mentioned the grounds on which it is believed that the higher operations of the mind are manifested through the agency of the grey matter of the convolutions. But, as the mind is capable of various actions, and various modes of action, it becomes natural to inquire whether the brain acts in all mental operations as a single organ, or as a congeries of many, each having a special function. This question is by no means of modern origin. It was believed by Galen that a relation exists between the form of the head and the mental vigour of the individual; and in the thirteenth century, Gordon, and since his time, Porta, Galleus, and others, made systematic endearours to associate special qualities of the mind with particular parts of the brain. In these early opinions may be recognised the elements of an imperfect doctrine of cranioscopy; but it was reserved for Gall to propound a more elaborate system, founded on extensive research, characterized by much originality, and rendered attractive by the peculiar genius and enthusiasm of 